\title{
Controlled release from a mechanically-stimulated thermosensitive self-heating composite hydrogel
}

\author{
Mohamadreza Nassajian Moghadam a, Vitaliy Kolesov ${ }^{\mathrm{b}}$, Arne Vogel a , Harm-Anton Klok ${ }^{\mathrm{b}}$, \\ Dominique P. Pioletti ${ }^{a, *}$ \\ a École Polytechnique Fédérale de Lausanne (EPFL), Institute of Bioengineering, Laboratory of Biomechanical Orthopedics, Switzerland \\ b École Polytechnique Fédérale de Lausanne (EPFL), Institut des Matériaux and Institut des Sciences et Ingénierie Chimiques, Laboratoire des Polymères, \\ Switzerland
}

\section{A R T I C L E I N F O}

\section{Article history:}

Received 21 August 2013

Accepted 17 September 2013

Available online 7 October 2013

\section{Keywords:}

Hydrogel

Controlled release

Mechanical loading

Viscous dissipation

Thermosensitive nanoparticles

\begin{abstract}
A B S T R A C T
Temperature has been extensively explored as a trigger to control the delivery of a payload from environment-sensitive polymers. The need for an external heat source only allows limited spatiotemporal control over the delivery process. We propose a new approach by using the dissipative properties of a hydrogel matrix as an internal heat source when the material is mechanically loaded. The system is comprised of a highly dissipative hydrogel matrix and thermo-sensitive nanoparticles that shrink upon an increase in temperature. Exposing the hydrogel to a cyclic mechanical loading for a period of $5 \mathrm{~min}$ leads to an increase of temperature of the nanoparticles. The concomitant decrease in the volume of the nanoparticles increases the permeability of the hydrogel network facilitating the release of its payload. As a proof-of-concept, we showed that the payload of the hydrogel is released after 5-8 min following the initiation of the mechanical loading. This delivery method would be particularly suited for the release of growth factor as it has been shown that cell receptor to growth factor is activated 5-20 min following a mechanical loading.
\end{abstract}

(c) 2013 Elsevier Ltd. All rights reserved.

\section{Introduction}

Most of the efforts in the field of drug delivery systems has focused on the development of environment-sensitive polymers. Temperature and $\mathrm{pH}$ are commonly used environmental variables [1-4]. While $\mathrm{pH}$ can be coupled to variations within the body, temperature sensitive polymers are designed to be altered either externally [5-7] or are in off/on mode almost immediately after being injected in the body [8-10].

Temperature-responsive drug delivery systems are usually based on polymer hydrogels with a lower critical solution temperature (LCST) of around $38{ }^{\circ} \mathrm{C}$. The drug is released when the tissue surrounding the hydrogel reaches a temperature slightly above normal body temperature $[4,11]$. While these systems work well for a number of applications, they also have some limitations. The need for an external means to cool or heat in many applications only allows limited spatiotemporal control over the delivery process.

\footnotetext{
* Corresponding author. EPFL/STI/IBI/LBO, Station 19, 1015 Lausanne, Switzerland. Tel.: +4121693 8341; fax: +41216938660.

E-mail address: dominique.pioletti@epfl.ch (D.P. Pioletti).
}

As hydrogels have dissipative properties, their temperature may also be altered internally by viscous dissipation during cyclic loading, a process generally referred to as self-heating [12-14]. There are potentially several advantages of using the self-heating property of materials for drug delivery.

First, the drug release is coupled to a mechanical loading. As mechanical loading has been demonstrated to activate some growth factor cell receptors involved in the healing process of different tissues such as cartilage [15-18], the coupling of mechanical loading and drug release could induce some positive synergetic effects. Drug delivery system coupled to mechanical loading has already been developed, the drug being simultaneously released during the mechanical loading [19-21].

However, it is important to realize that cell receptors are not immediately activated following a mechanical loading. A delay of 5-20 min has been observed between the initiation of the mechanical loading and the activation of the cell receptor [22]. To induce a maximum potency, the release of a drug following a mechanical loading should then also be delayed by several minutes.

As the self-heating property induces a local temperature increase, which is related to the dissipative properties of the material and to the number of loading cycles, a delay can be obtained between the initiation of the mechanical loading and the temperature 
increase triggering the drug release. The use of dissipative energy generated in hydrogel could then offer a second advantage, which is the unprecedented spatiotemporal control over the delivery process. In particular, the use of self-heating property would be particularly suited for the delivery of growth factors to induce healing in a cartilage defect where the cells are naturally subjected to mechanical loading.

In this study, we establish the proof-of-concept that dissipative properties can be used as a new environmental variable to spatiotemporally control the release of a drug.

\section{Materials and methods}

2.1. Principle of dissipation used as an environmental variable

To explore the feasibility of using dissipative properties to control delivery from thermosensitive polymer based systems, we developed a unique hydrogel system consisting of two components: i) poly(2-hydroxyethyl methacrylate) (PHEMA)based hydrogel matrix with highly dissipative properties and ii) poly(N-isopropyl acrylamide) (PNIPAM)-based thermosensitive nanoparticles, which are entrapped in the matrix hydrogel and shrink at temperatures above their LCST
The principle used to temporally control the delivery of a payload following a mechanical loading is shown in Fig. 1A. Upon applying a mechanical load, a part of the mechanical energy is transformed into heat due to the dissipative properties of the hydrogel matrix. The heat produced by the hydrogel increases the temperature of the nanoparticles above their LCST and induces their collapse, which subsequently facilitates diffusion of the payload outside the hydrogel. The dissipative properties of the hydrogel can be modulated to link the duration of the cyclic loading with a targeted increase in temperature. Thus, a specific delay between the initiation of the mechanical stimulation and the release of a payload can be obtained.

HEMA is a hydrogel-forming material that is widely used in the biomaterials field $[23,24]$. Water content and crosslink density are the key parameters to control the mechanical and dissipative properties of these hydrogels [25,26]. The hydrogels can be obtained using different crosslinkers in a one-step photo-polymerization process [27]. The PHEMA hydrogels investigated in this study were crosslinked with ethylene glycol dimethacrylate (EGDMA) and contained $40 \%$ water

\subsection{Materials}

2,2-dimethoxy-2-phenylacetophenone (Irgacure 651, 97\%), ethylene glycol dimethacrylate (EGDMA, 98\%), TWEEN ${ }^{\circledR} 80$, Span $^{\circledR} 80, \mathrm{~N}, \mathrm{~N}, \mathrm{~N}^{\prime}, \mathrm{N}^{\prime}$-Tetramethylethylenediamine (TMEDA, 99\%), Ammonium persulfate ( $\geq 98 \%$ ) and Xylene Cyanole FF were purchased from Aldrich and used as received. 2-Hydroxyethyl methacrylate (HEMA, 97\%) and poly(ethylene glycol) dimethacrylate (PEGDMA, average $M_{\mathrm{n}} 550$ ) were purchased from Aldrich and purified by basic aluminum oxide column chromatography to remove inhibitor. $\mathrm{N}$-isopropylacrylamide (Aldrich, $97 \%$ ) was purified
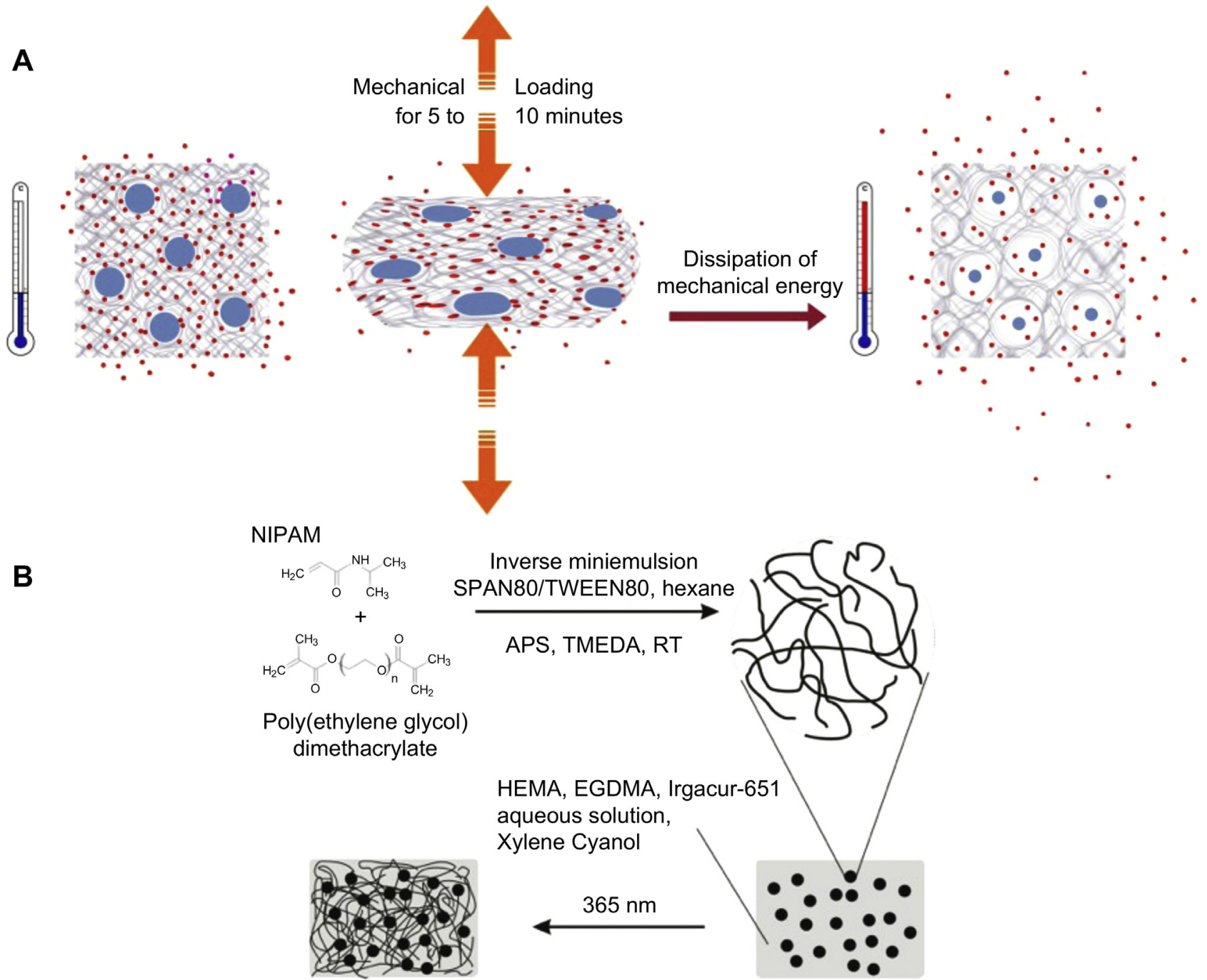

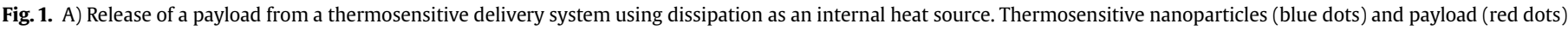

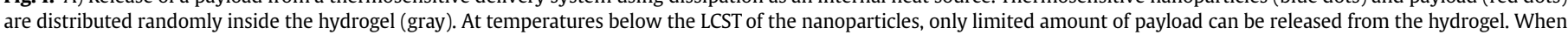

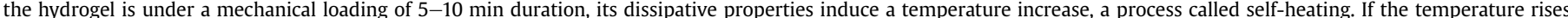

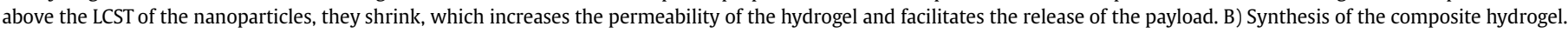
(For interpretation of the references to color in this figure legend, the reader is referred to the web version of this article.) 
by recrystallization from hexane. Spectra/Por 6 dialysis tubing, 10K MWCO was purchased from Spectrum Labs Europe.

\subsection{Preparation of the nanoparticles}

The organic phase consisted of $\mathrm{n}$-hexane $(15 \mathrm{~mL})$ and a mixture of surfactants TWEEN80 (75 mg) and SPAN80 (225 mg). The oxygen dissolved in the organic phase was removed by evacuating the surfactant mixture for 5 min followed by purging with nitrogen for short period (repeated 3 times). Hexane was purged with nitrogen for $10 \mathrm{~min}$. The aqueous phase was prepared as follows: $150 \mathrm{mg}$ of NIPAM were added to the flask along with $10 \mathrm{mg}$ of poly(ethylene glycol)dimethacrylate (PEGDMA). Solid monomer and cross-linker were dissolved in $0.4 \mathrm{~mL}$ PBS pH 7.4 and sonicated. Organic phase was added to aqueous phase and the mixture was emulsified by sonicating $30 \mathrm{~s}$. Polymerization was initiated by the addition of 2 drops of tetramethylethylenediamine and $25 \mathrm{mg}$ of ammonium persulfate in $0.1 \mathrm{~mL} \mathrm{PBS} \mathrm{pH}$ 7.4 during sonication. Additional drops of TMEDA were added $10 \mathrm{~min}$ after; the miniemulsion was stirred for $4 \mathrm{~h}$ at room temperature. The reaction was stopped by exposure to air. Hexane was removed on rotary evaporator at $30^{\circ} \mathrm{C}$ and $5 \mathrm{mbar}$, waxlike substance was redispersed in ethyl ether and precipitated. Supernatant was removed, and particles were redispersed in ether two more times. After that, the particles were redispersed in water, dialyzed against water for 3 days (MWCO 10,000 membrane, water was changed at least twice a day) and freeze-dried. Purified particles were redispersed in water, and the diameter was analyzed by DLS as the average of 10 measurements.

\subsection{Preparation of the composite hydrogel}

2-Hydroxyethyl methacrylate (HEMA) $(880 \mu \mathrm{L})$, ethylene glycol dimethacrylate (EGDMA) $(84 \mu \mathrm{l}, 6 \% \mathrm{~mol})$, and Xylene Cyanol FF aqueous solution $(1 \mathrm{mg} / \mathrm{ml}, 590 \mu \mathrm{L})$ were mixed in a $5 \mathrm{~mL}$ Eppendorf tube. The Xylene Cyanol FF was used as drug model. For hydrogels containing nanoparticles, the aqueous phase also contained $15 \mathrm{mg} / \mathrm{mL}$ nanoparticles, which is equal to $5.5 \mathrm{mg} / \mathrm{mL}$ nanoparticles in total reaction mixture. A solution of Irgacur-651 (photo initiator) in ethanol ( $36 \mu \mathrm{L}$ of $57 \mathrm{mg} / \mathrm{mL}$ solution) was then added to the polymer mixture. The mixture was stirred and sonicated for $1 \mathrm{~min}$, then transferred to the cylindrical wells ( $8 \mathrm{~mm}$ diameter and $4 \mathrm{~mm}$ depth), placed under UV lamp (365 nm, 8 Watt) and irradiated for $15 \mathrm{~min}$, whilst maintaining the temperature below $25{ }^{\circ} \mathrm{C}$ (using a flow of compressed air). The samples were washed and kept in $1 \mathrm{~mL}$ water for two days before mechanical tests (Fig. 1B).

\subsection{Quantification of Xylene Cyanole FF release}

After each test the supernatant water was collected from the test chamber and the amount of released Xylene Cyanole FF was quantified spectrophotometrically at $590 \mathrm{~nm}$. The release amount $c_{\mathrm{d}}(t)$ was reported as $\mu \mathrm{g} / \mathrm{mL}$ using the normalized curves we prepared by spectrophotometric analysis of solutions at different concentrations of Xylene Cyanol FF in water.

\subsection{Mechanical loading}

Mechanical compressive test was performed with an Instron E3000 linear mechanical testing machine (Norwood, MA, USA). To provide a heat-isolated environment, controlling the initial temperature and monitoring the temperature during the test, a specifically designed thermally isolated system was installed on the mechanical testing machine (Fig. 1S, Supplementary data). The temperature of the test chamber was maintained by water circulation around the chamber either at $34{ }^{\circ} \mathrm{C}$ or at $36{ }^{\circ} \mathrm{C}$ for different experiments. The hydrogel immersed in $600 \mu \mathrm{L}$ fresh water was placed in the center of the chamber. The hydrogel temperature was monitored with a thermistor positioned in the center of test chamber (Fig. 1S, Supplementary data). Cyclic compression was applied at $1.5 \mathrm{~Hz}$ on displacement control mode. Deformations of $15 \%$ amplitude following a 5\% prestrain were applied for 5 and $8 \mathrm{~min}$. The temperature was recorded every $10 \mathrm{~s}$ during the test.

\subsection{Effective diffusion coefficient measurement}

To calculate the effective diffusion coefficient of the composite hydrogel, we prepared samples in the form of microfilms by injecting the polymer mixture between two glass slides having a $150 \mu \mathrm{m}$ gap and polymerized them under UV light. After polymerization, films were punched with $8 \mathrm{~mm}$ punch and immersed in $600 \mu \mathrm{L}$ water. Hydrogels with or without nanoparticles were placed either at $34^{\circ} \mathrm{C}$ or at $39{ }^{\circ} \mathrm{C}$ (6 samples per group). Xylene Cyanole FF release $\left(c_{\mathrm{d}}(t)\right)$ was measured every $2 \mathrm{~h}$ for $14 \mathrm{~h}$ from each of the 4 different hydrogel groups (with or without nanoparticles, at $34^{\circ} \mathrm{C}$ and $39^{\circ} \mathrm{C}$ ). The transient distribution of Xylene Cyanole FF in a cylindrical sample is governed by the following equation [28]:

$\frac{\partial c}{\partial t}=\frac{D_{\mathrm{eff}}}{\phi}\left(\frac{1}{r} \frac{\partial}{\partial r}\left(r \frac{\partial c}{\partial r}\right)+\frac{\partial^{2} c}{\partial z^{2}}\right)$

where $c$ is the Xylene Cyanole FF concentration within the sample, $D_{\text {eff }}$ is the effective diffusivity and $\phi$ is the hydrogel water content equal to $40 \%$ in our case.

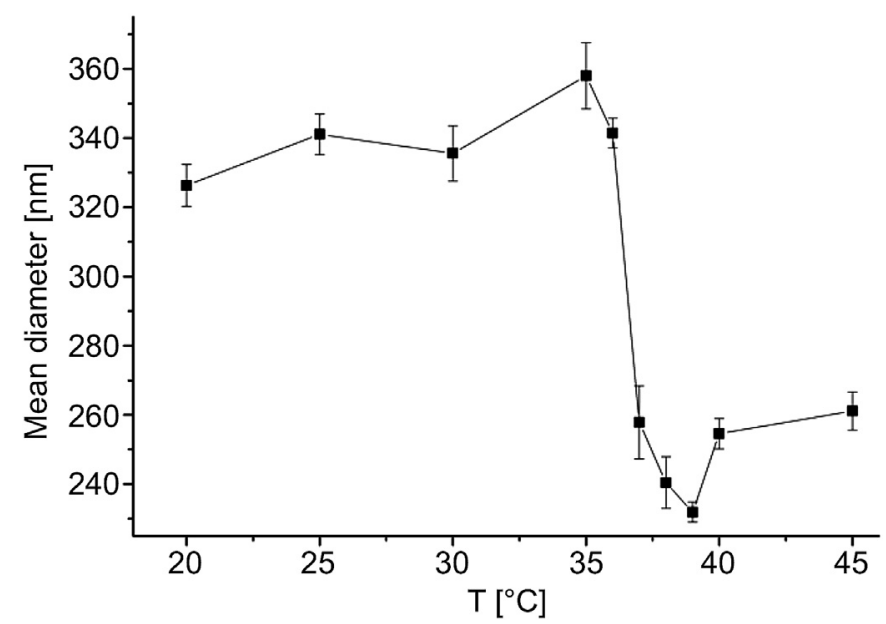

Fig. 2. The effect of temperature on the size of the PNIPAM nanoparticles. The temperature leading to decrease in nanoparticle size is referred to as LCST and is comprised between 36 and $37^{\circ} \mathrm{C}$. The diameter of the nanoparticles was analyzed by DLS as the average of 10 measurements.

Considering the boundary condition $c=0$ at $r=R, z=0$ and $z=h$ (R and h: sample diameter and height) and the initial condition $c=c_{0}$ (initial Xylene Cyanole FF concentration inside the hydrogel), we obtained the analytical solution for $c$. By integrating $c$ over the sample fluid volume and subtracting the result from the total Xylene Cyanole FF release gives the following solution for the Xylene release in the water bath $c_{\mathrm{d}}(t)$ :

$c_{\mathrm{d}}(t)=c_{\max }\left[1-\sum_{n=1}^{\infty} \sum_{m=1}^{\infty} \frac{16\left[1-(-1)^{n}\right]}{q_{m}^{2} n^{2} \pi^{2}} \exp \left(-\frac{D_{\mathrm{eff}} \lambda_{\mathrm{nm}}^{2} t}{\phi}\right)\right]$

$c_{\mathrm{d}}(t)$ is the Xylene Cyanole FF release, $c_{\max }$ is the total Xylene Cyanole FF release from the sample in water if we assume all Xylene in the sample is released in $600 \mu \mathrm{L}$ (for microfilm samples $\left.c_{\max }=2.83 \mu \mathrm{g} / \mathrm{mL}\right), q_{\mathrm{m}}$ is the $\mathrm{m}^{\text {th }}$ zero of zero-order Bessel function $\left(J_{0}\right)$ and

$\lambda_{\mathrm{nm}}^{2}=\frac{q_{\mathrm{m}}^{2}}{R^{2}}+\frac{n^{2} \pi^{2}}{h^{2}}$

All parameters in Equation (2) except $D_{\text {eff }}$ were known or measured independently. $D_{\text {eff }}$ was calculated by fitting Equation (2) to the experimental data from desorption bath (Fig. 2S, Supplementary data).

\section{Results}

\subsection{Temperature response of nanoparticles}

The nanoparticles had a diameter of $340 \mathrm{~nm}$ as determined by dynamic light scattering (DLS) at room temperature and showed a lower critical solution temperature of $37{ }^{\circ} \mathrm{C}$ (Fig. 2). At $37{ }^{\circ} \mathrm{C}$, the PNIPAM nanoparticles collapsed and had an average diameter of $255 \mathrm{~nm}$. The resulting decrease in volume corresponds to about $50 \%$.

\subsection{Composite hydrogels}

The final formulation of the composite hydrogel consists of an EGMDA crosslinked PHEMA-based hydrogel containing 40\% aqueous phase with dispersed nanoparticles and Xylene Cyanole FF, which was used as model drug. Fig. 3 shows the dispersion of nanoparticles in hydrogels with different concentrations of nanoparticles. For the final formulation we had $5.5 \mathrm{mg}$ nanoparticles per $1000 \mu \mathrm{L}$ of reaction mixture.

\subsection{Self-heating of hydrogels due to dissipation}

The self-heating property of the composite hydrogels were investigated using a custom-designed thermally isolated system, which was coupled to a mechanical testing machine (Fig. 1S). To 


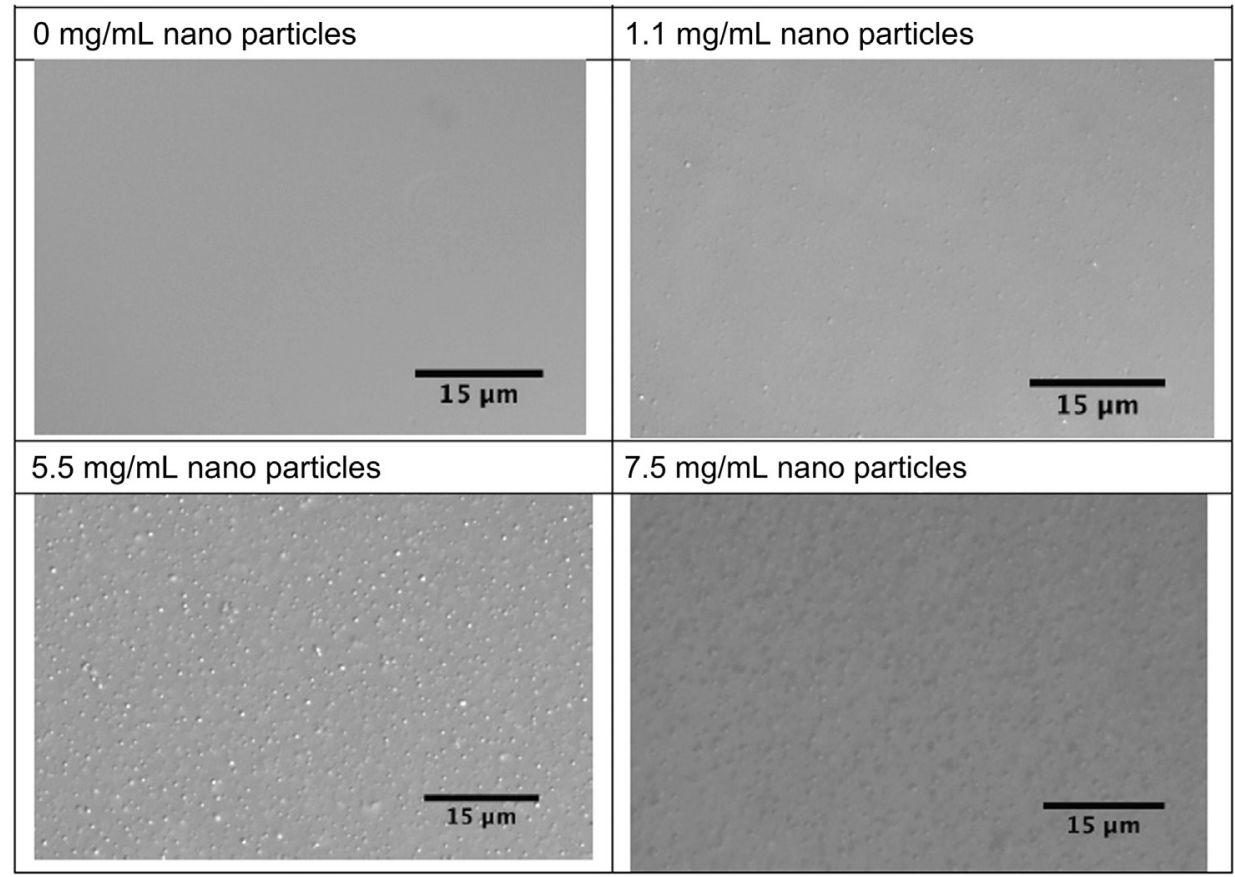

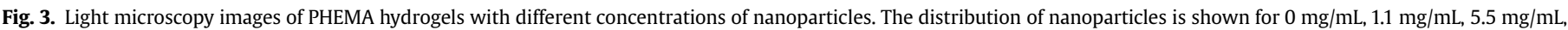
and $7.5 \mathrm{mg} / \mathrm{mL}$ of nanoparticles concentration in the reaction mixture before polymerization. The $5.5 \mathrm{mg} / \mathrm{mL}$ nanoparticles concentration was used for the experiments.

simulate human body environment, the initial temperature of the thermally isolated system was set to $36^{\circ} \mathrm{C}$. It was verified that after 5 min of loading ( $5 \%$ of prestrain followed by a $15 \%$ deformation amplitude at $1.5 \mathrm{~Hz}$ ) the temperature of the hydrogel reached $37^{\circ} \mathrm{C}$ (Fig. 4A). In another experiment, we set the environment temperature to $34{ }^{\circ} \mathrm{C}$ and after $5 \mathrm{~min}$ loading the temperature reached $34.7^{\circ} \mathrm{C}$ (Fig. 4B).

\subsection{Drug release due to dissipation}

Under mechanical loading, when environmental temperature was fixed to $36{ }^{\circ} \mathrm{C}$, a statistically significant increase (133\%) in Xylene Cyanole FF release was observed between the 5 and $8 \mathrm{~min}$ loading demonstrating the delayed release of the dye following a mechanical loading (Fig. 5A). To rule out the possibility that the mechanical loading directly induced the expulsion of the Xylene Cyanole FF out of the hydrogel, we repeated the experiment but setting the initial temperature of the thermally isolated system to $34{ }^{\circ} \mathrm{C}$. In this test, the temperature of the hydrogel following the mechanical stimulation stayed below the LCST of nanoparticles (Fig. 4B). It can be observed from Fig. 5A, that when the hydrogel temperature is below the LCST of nanoparticles, there was no significant difference in Xylene release between 5 or 8 min loading. Finally to confirm the role of the nanoparticles in the observed phenomenon and to exclude that the observed effects were due to a direct thermal effect on the hydrogel, the tests at $34{ }^{\circ} \mathrm{C}$ and $36{ }^{\circ} \mathrm{C}$ were repeated with hydrogels that did not contain the thermosensitive nanoparticles. Results in Fig. 5B showed that only the combination of nanoparticles in hydrogels and mechanical loading inducing a temperature increase of the hydrogel above $37{ }^{\circ} \mathrm{C}$ allowed a statistically significant increase of the release of Xylene Cyanole FF.

\subsection{Effective diffusion coefficient}

When nanoparticles are incorporated in the hydrogels and the hydrogel temperature is above the LCST of the nanoparticles, the effective diffusivity of the hydrogel increases by a factor 3 (Fig. 6). When the hydrogel contained no nanoparticles, the effective diffusivity of the hydrogel was not affected by the temperature increase.
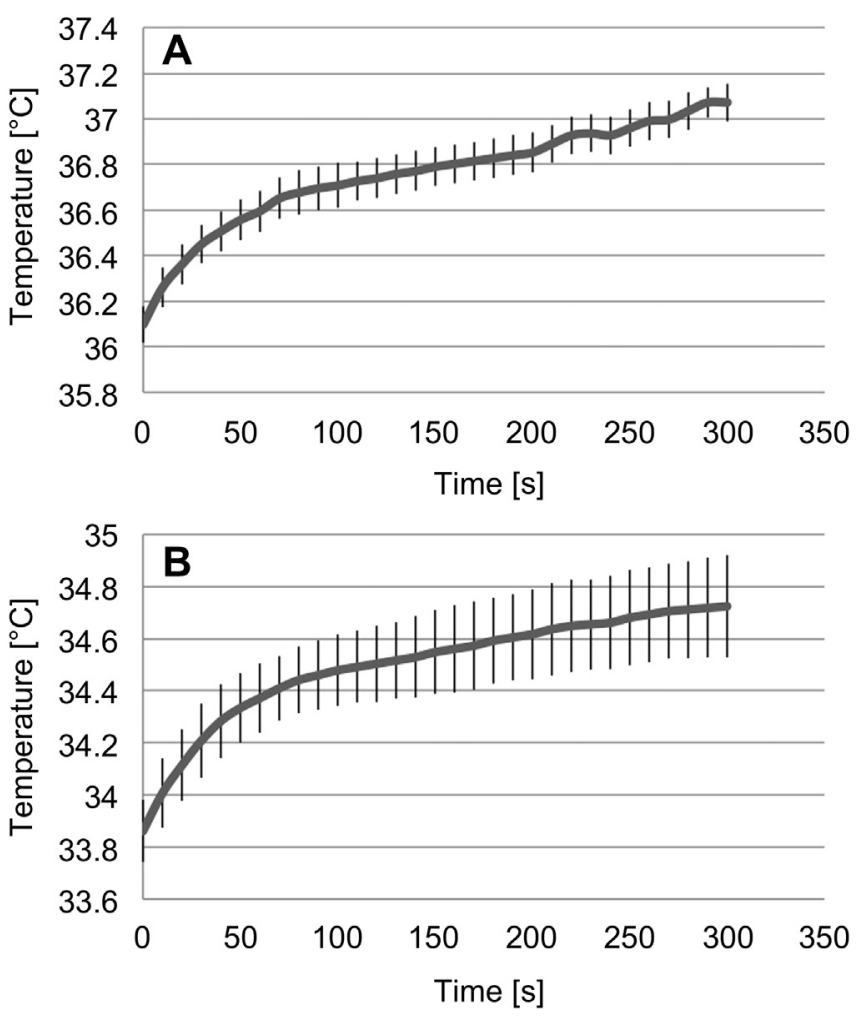

Fig. 4. Self-heating of the hydrogel following cyclic compressive load. The initial temperature was set in the test chamber (Fig. 1S) and increased due to the dissipated heat. A: The initial temperature was set to $36^{\circ} \mathrm{C}$ and increased to $37^{\circ} \mathrm{C}$ after $5 \mathrm{~min}$ of loading. B: The initial temperature was set to $34^{\circ} \mathrm{C}$ and increased to $34.7^{\circ} \mathrm{C}$ after $5 \mathrm{~min}$ of loading. 

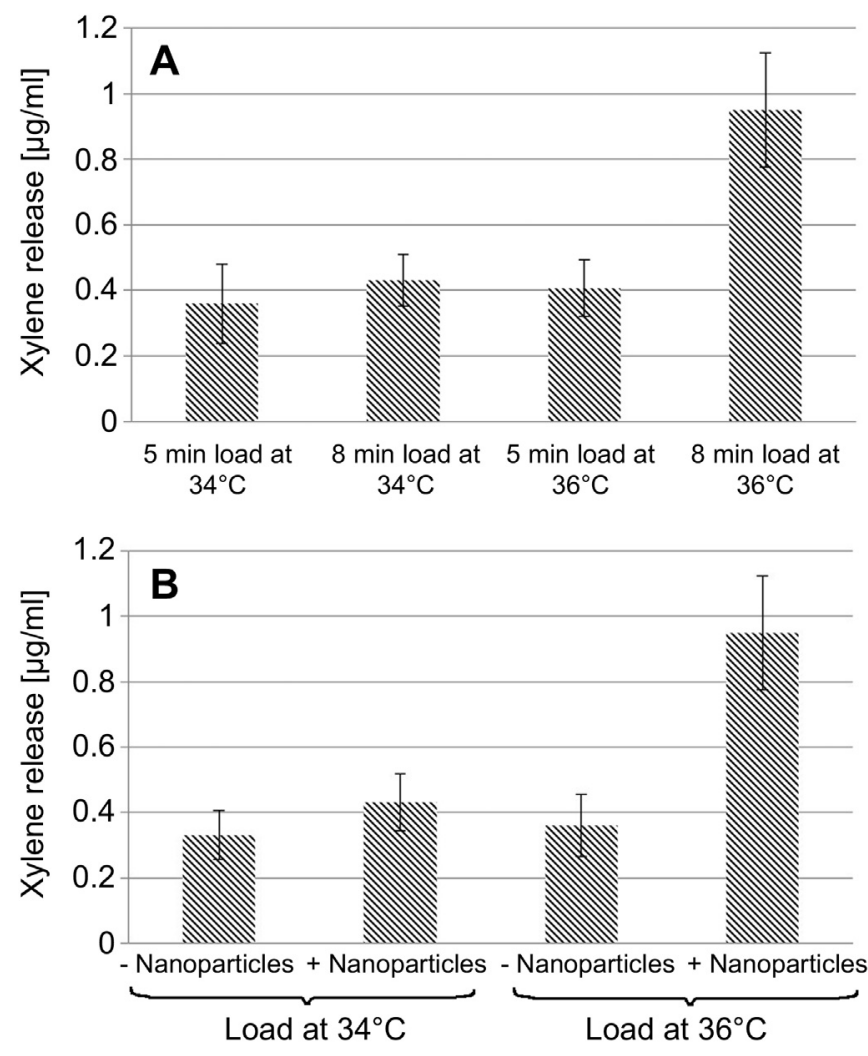

Fig. 5. A) Temporal control of Xylene Cyanole FF release triggered by dissipation properties of the composite hydrogel. The release of the Xylene Cyanole FF from hydrogels containing nanoparticles was quantified after 5 and $8 \mathrm{~min}$ of cyclic mechanical loading. When the initial temperature was set at $34^{\circ} \mathrm{C}$, no statistical difference was observed for the Xylene release between 5 or $8 \mathrm{~min}$. When the initial temperature was set to $36{ }^{\circ} \mathrm{C}$, a statistically significant increase $(133 \%)$ in Xylene Cyanole FF release was measured between the 5 and 8 min loading ( $p=0.026, T$-test type 1, 1 tailed). B) Effect of nanoparticles on Xylene Cyanole FF release during mechanical loading. Mechanical load was applied on hydrogels with and without nanoparticles for $8 \mathrm{~min}$ at the initial temperature of $34{ }^{\circ} \mathrm{C}$ and $36{ }^{\circ} \mathrm{C}$. No statistically significant increase in Xylene release was observed at $36{ }^{\circ} \mathrm{C}$ for hydrogels without nanoparticles. A statistically significant increase (125\%) of dye release was measured when the load was applied on hydrogels containing nanoparticles and initially thermostated to $36{ }^{\circ} \mathrm{C}(p=0.031, T$-test type 1,1 tailed $)$.

\section{Discussion}

Mechanical loading has been demonstrated to activate growth factor receptors of cells involved in the healing process of different tissues $[15,16,18]$. However, a delay is necessary for the activation of

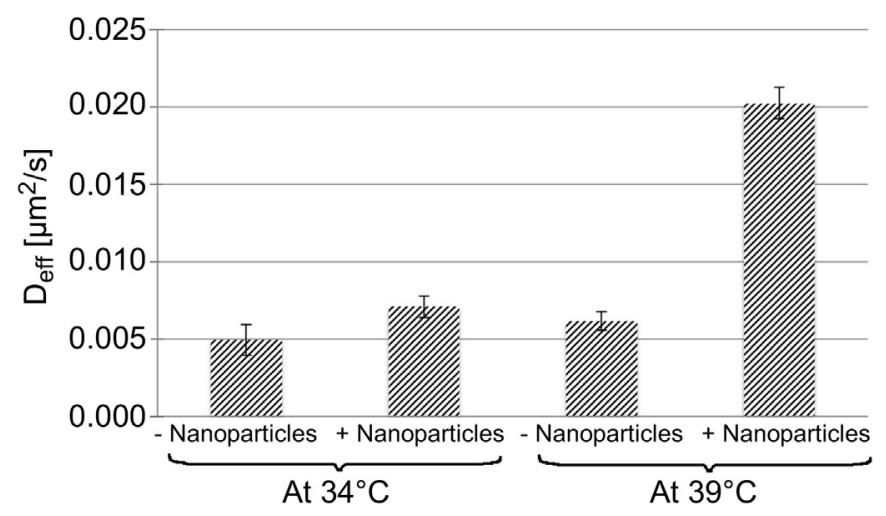

Fig. 6. Thin PHEMA hydrogel film effective diffusion coefficient $\left(D_{\text {eff }}\right)$. $D_{\text {eff }}$ was calculated by curve fitting of the mathematical model for Xylene Cyanole FF release from microfilm hydrogel with or without nanoparticles at temperature above and below the LCST of the nanoparticles. cell receptors following the initiation of a mechanical stimulation [22]. A synergetic effect combining the delivery of a drug and the mechanically induced activation of the corresponding receptor could be maximized if drug release is delayed by several minutes following the initiation of the mechanical stimulation. In this study we proposed to use the dissipative property of hydrogel as a new environmental variable allowing to spatially and temporally control the release of a drug. The proposed delivery system could then not only provide unparalleled spatiotemporal control over the release process, but also allow the delivery to be synchronized with the mechanical stimulus favoring the healing of the damaged tissue. The dissipative properties of the hydrogel can be modulated to link the duration of the cyclic loading with a targeted increase in temperature. Thus, a specific delay between the initiation of the mechanical stimulation and the release of a payload can be obtained.

The most probable effect which can explain the results presented in Fig. 5A and B is to consider that the temperature-induced shrinkage of nanoparticles due to the dissipation properties of the hydrogel changed the permeability of the hydrogel. To verify this claim, the effective diffusion coefficient was calculated from Xylene Cyanole FF release experiments performed on thin layer hydrogel film. The obtained result confirmed that the augmentation of the hydrogel temperature affects its permeability. By itself, the observation that a cyclic mechanical loading can affect hydrogel permeability is an original result not previously reported. As the change in permeability is obtained following the temperature increase, which in turn is due to the mechanical loading, a time delay is obtained between the initiation of the mechanical loading and the release of the payload. The value of this time delay can be tuned by varying the LCST of nanoparticles $[4,29]$ and/or the dissipative properties of the hydrogel as well as the loading amplitude and frequency.

As previously mentioned, different systems have been developed to couple mechanical loading and drug release [19-21]. However, most of these systems could be considered as a sponge containing a payload so that no specific correlation between mechanical loading and drug release could be controlled. Another advantage of the proposed system is that it considerably reduced undesirable passive drug release. If we measure the amount of passive release over days from the composite hydrogel (Fig. 2S, Supplementary data), we observe that after 5 days its value represents $9 \%$ of total loaded payload if the temperature is above the LCST and less than $4 \%$ of total loaded Xylene if the temperature is below the LCST. In comparison, the release due to mechanical load was about $1.2 \%$ of total Xylene after $8 \mathrm{~min}$. The reluctancy of the system to passive release is most probably due to the hydrophobic properties of PHEMA-based hydrogels. In spite of hydrophilic nature of HEMA monomers, it is postulated that PHEMA hydrogel has, in addition to its covalently linked network structure, a secondary structure stabilized by hydrophobic bonding [30]. It has been showed that for a hydrophobic porous material, because of an defiltration (drainage) and infiltration pressure, force is required to move liquid in and out of the material pores [31]. Without mechanical loading the structure is reluctant to exchange fluid and facilitate the payload release. Therefore the amount of passive release is highly decreased with the developed composite hydrogel.

High dissipation properties of PHEMA hydrogels also increase its toughness, which is important if the composite hydrogel is used at load-bearing sites like in cartilage. In general because hydrogels have poor mechanical properties and are very brittle, their use is limited for load-bearing applications. It has been shown that the poor mechanical performance and fragility of hydrogels mainly originate from their very low resistance to crack propagation due to the lack of an efficient energy dissipation mechanism in the gel network $[32,33]$. The high dissipation property of the developed 
composite hydrogel is then a particular advantage also for its mechanical behavior.

\section{Conclusion}

Temporal control over the delivery process induced by a mechanical loading is proposed in this study and is an innovative way to deliver a payload. Dissipation properties of materials can be considered as a new environmental variable to trigger the release from thermosensitive, polymer-based delivery systems. A synergetic effect between loading and delayed payload delivery could be obtained with the use of dissipative phenomenon, which is not possible with other delivery systems.

\section{Acknowledgments}

This work was supported by the Swiss National Science Foundation (\#406240_126070, PNR 62 program) and the InterInstitutional Center for Translational Biomechanics EPFL-CHUVDAL. We thank Marion Brun from the CHUV-Lausanne for the drawing of Fig. $1 \mathrm{~A}$.

\section{Appendix A. Supplementary data}

Supplementary data related to this article can be found online at http://dx.doi.org/10.1016/j.biomaterials.2013.09.065.

\section{References}

[1] Gil ES, Hudson SM. Stimuli-responsive polymers and their bioconjugates. Prog Polym Sci 2004;29:1173-222.

[2] Gupta P, Vermani K, Garg S. Hydrogels: from controlled release to $\mathrm{pH}-$ responsive drug delivery. Drug Discov Today 2002;7:569-79.

[3] Klouda L, Mikos AG. Thermoresponsive hydrogels in biomedical applications. Eur J Pharm Biopharm 2008;68:34-45.

[4] Qiu Y, Park K. Environment-sensitive hydrogels for drug delivery. Adv Drug Deliv Rev 2012;64:49-60.

[5] Hoare T, Santamaria J, Goya GF, Irusta S, Lin D, Lau S, et al. A magnetically triggered composite membrane for on-demand drug delivery. Nano Lett 2009:9:3651-7.

[6] Schmaljohann D. Thermo- and pH-responsive polymers in drug delivery. Adv Drug Deliv Rev 2006;58:1655-70.

[7] Yavuz MS, Cheng YY, Chen JY, Cobley CM, Zhang O, Rycenga M, et al. Gold nanocages covered by smart polymers for controlled release with nearinfrared light. Nat Mater 2009;8:935-9.

[8] Gong C, Qi T, Wei X, Qu Y, Wu Q Luo F, et al. Thermosensitive polymeric hydrogels as drug delivery systems. Curr Med Chem 2013:20:79-94.

[9] Jeong B, Kim SW, Bae YH. Thermosensitive sol-gel reversible hydrogels. Adv Drug Deliv Rev 2002;54:37-51.

[10] Yu L, Ding JD. Injectable hydrogels as unique biomedical materials. Chem Soc Rev 2008;37:1473-81.

[11] Zhang J, Misra RDK. Magnetic drug-targeting carrier encapsulated with thermosensitive smart polymer: core-shell nanoparticle carrier and drug release response. Acta Biomater 2007:3:838-50.
[12] Ahearne M, Yang Y, El Haj AJ, Then KY, Liu KK. Characterizing the viscoelastic properties of thin hydrogel-based constructs for tissue engineering applications. J R Soc Interface 2005;2:455-63.

[13] Karnaukhov VG, Yakovlev GA, Goncharov LP. Self-heating of viscoelastic materials under cyclic loads. Strength Mater 1975;7:164-8.

[14] Schapery RA. Effect of cyclic loading on the temperature in viscoelastic media with variable properties. Aiaa J 1964;2:827-35.

[15] Hiyama A, Mochida J, Iwashina T, Omi H, Watanabe T, Serigano K, et al, Synergistic effect of low-intensity pulsed ultrasound on growth factor stimulation of nucleus pulposus cells. J Orthop Res 2007;25:1574-81.

[16] Discher DE, Mooney DJ, Zandstra PW. Growth factors, matrices, and forces combine and control stem cells. Science 2009;324:1673-7.

[17] Mauck RL, Nicoll SB, Seyhan SL, Ateshian GA, Hung CT. Synergistic action of growth factors and dynamic loading for articular cartilage tissue engineering. Tissue Eng 2003;9:597-611.

[18] Neu CP, Khalafi A, Komvopoulos K, Schmid TM, Reddi AH. Mechanotransduction of bovine articular cartilage superficial zone protein by transforming growth factor beta signaling. Arthritis Rheum 2007;56:3706-14.

[19] Lee KY, Peters MC, Anderson KW, Mooney DJ. Controlled growth factor release from synthetic extracellular matrices. Nature 2000;408:998-1000.

[20] Edelman ER, Fiorino A, Grodzinsky A, Langer R. Mechanical deformation of polymer matrix controlled release devices modulates drug release. J Biomed Mater Res 1992;26:1619-31.

[21] Korin N, Kanapathipillai M, Matthews BD, Crescente M, Brill A, Mammoto T, et al. Shear-activated nanotherapeutics for drug targeting to obstructed blood vessels. Science 2012;337:738-42.

[22] Tschumperlin DJ, Dai G, Maly IV, Kikuchi T, Laiho LH, McVittie AK, et al. Mechanotransduction through growth-factor shedding into the extracellular space. Nature 2004;429:83-6.

[23] Chirila TV. An overview of the development of artificial corneas with porous skirts and the use of PHEMA for such an application. Biomaterials 2001;22: $3311-7$.

[24] Dziubla TD, Torjman MC, Joseph JI, Murphy-Tatum M, Lowman AM. Evaluation of porous networks of poly(2-hydroxyethyl methacrylate) as interfacial drug delivery devices. Biomaterials 2001;22:2893-9.

[25] Lai YC, Quinn ET. The effects of initiator and diluent on the photopolymerization of 2-hydroxyethyl methacrylate and on properties of hydrogels obtained. Am Chem Soc Symp Ser 1997;673:35-50.

[26] Mabilleau G, Stancu IC, Honore T, Legeay G, Cincu C, Basle MF, et al. Effects of the length of crosslink chain on poly(2-hydroxyethyl methacrylate) (pHEMA) swelling and biomechanical properties. J Biomed Mater Res A 2006;77:35-42.

[27] Baker MV, Brown DH, Casadio YS, Chirila TV. The preparation of poly(2hydroxyethyl methacrylate) and poly \{(2-hydroxyethyl methacrylate)-co[poly(ethylene glycol) methyl ether methacrylate]\} by photoinitiated polymerisation-induced phase separation in water. Polymer 2009;50: $5918-27$.

[28] Quinn TM, Kocian P, Meister J- J. Static compression is associated with decreased diffusivity of dextrans in cartilage explants. Arch Biochem Biophys 2000;384:327-34

[29] Feil H, Bae YH, Feijen J, Kim SW. Effect of comonomer hydrophilicity and ionization on the lower critical solution temperature of $\mathrm{N}$-isopropylacrylamide copolymers. Macromolecules 1993;26:2496-500.

[30] Refojo MF. Hydrophobic interaction in poly(2-hydroxyethyl methacrylate) homogeneous hydrogel. J Polym Sci Part A 1967:5:3103-13.

[31] Zhao J, Culligan PJ, Germaine JT, Chen X. Experimental study on energy dissipation of electrolytes in nanopores. Langmuir 2009;25:12687-96.

[32] Abdurrahmanoglu S, Can V, Okay O. Design of high-toughness polyacrylamide hydrogels by hydrophobic modification. Polymer 2009;50:5449-55.

[33] Tuncaboylu DC, Sari M, Oppermann W, Okay O. Tough and self-healing hydrogels formed via hydrophobic interactions. Macromolecules 2011;44: 4997-5005. 